\title{
Archaeoastronomical Study of the Main Pyramids of Giza, Egypt: Possible Correlations with the Stars?
}

\author{
Vincenzo Orofino, Paolo Bernardini \\ Dipartimento di Matematica e Fisica “E. De Giorgi”, Università del Salento, Lecce, Italy \\ Email: vincenzo.orofino@unisalento.it
}

Received 23 October 2015; accepted 21 November 2015; published 24 November 2015

Copyright (C) 2016 by authors and Scientific Research Publishing Inc.

This work is licensed under the Creative Commons Attribution International License (CC BY). http://creativecommons.org/licenses/by/4.0/

\section{(c) (i) Open Access}

\begin{abstract}
Since long time various qualitative speculations have been proposed about the link between the three major Giza pyramids and the stars. In particular, according to a popular and controversial hypothesis (the so-called Orion Correlation Theory), a perfect coincidence would exist between the mutual positions of the three stars of the Orion Belt and those of the main Giza pyramids. In the present paper, this apparent coincidence has been subjected to some statistical verifications, in order to assess the probability that the correlation between stars and pyramids, both in relative position and in luminosity/height, can be merely due to the case. These statistical analyses have been performed by means of Monte Carlo simulations and have been coupled with previous astronomical/astrophysical tests of the presumed correlation, finding that the coincidence does not seem to be fortuitous and that it is compatible with the naked-eye astrometry and photometry of the Orion Belt stars. On the contrary, unlike what stated by another popular and controversial theory (the so-called Cygnus-Giza Correlation), we have found no coincidence between the mutual positions of the three pyramids and those of the three stars of the short arm of the asterism of Northern Cross, in the Cygnus constellation.
\end{abstract}

\section{Keywords}

Egypt, Old Kingdom, Pyramids, Archaeoastronomy, Statistics

\section{Introduction: The Orion Belt and the Egyptian Astronomy}

The interest of the ancient Egyptians in the celestial phenomena is suggested by various inscriptions found out on the sarcophagi of the Middle Kingdom (1990-1780 BC) and in the tombs of the New Kingdom (1530-1080 
BC), such as the famous tomb of Senmut, a dignitary of the queen Hatshepsut (ca. XV century BC).

Furthermore, it is now sure that the ancient Egyptians orientated their monuments and sacred buildings toward the rising points of some bright stars, such as Sirius and Canopus, and that they used other stars (namely those of the constellation Ursa Major, or the Big Bear) to align temples and pyramids with the cardinal points (see Belmonte, Shaltout, \& Fekri, 2008, and references therein).

It was well documented that, as early as the Middle Kingdom, the Egyptian astronomers were able to track the movements (recording the times of rising, culmination, setting, the period of invisibility and so on) of a set of 36 stars, or small groups of stars, called “decans”, mainly used for time keeping (Magli, 2006).

However, even if compelling evidence of the observation of decans existed only in the archaeological finds of the Middle Kingdom or later, according to Magli (2009a), it was not unreasonable to suppose that such observations could have their roots in much more ancient astronomical practices dating back to the Old Kingdom (2700-2200 BC). Actually, as early as the Second Dynasty (2650 BC) the High Priest of the sanctuary of Heliopolis was called the "Chief of the Observers", and this testified that during the Old Kingdom astronomical observations were surely one of the main duties of some Egyptian priests (Magli, 2009a). In addition, during this early period, Egyptians knew the five planets Mercury, Venus, Mars, Jupiter and Saturn, due to their movement with respect to the "fixed" stars.

Among all the decans, a very important role was played, in addition to Sirius ( $\alpha$ Canis Majoris), also by the stars of the Orion Belt, a linear asterism of three evenly spaced objects in which the northernmost star, Mintaka (or $\delta$ Orionis), is slightly out of the axis connecting the southernmost object, Alnitak (or $\zeta$ Orionis), to the central one, Alnilam (or $\varepsilon$ Orionis). Even if alternative interpretations exist (Baux, 1994; Legon, 1995), it was commonly thought that the ancient Egyptians associated the Orion constellation (Sah), and in particular the Orion Belt, to Osiris, one of the most important gods of the Egyptian Pantheon, while the star Sirius (Sopdet or Sothis) represented the goddess Isis, sister and wife of Osiris (Bauval, 2006).

\section{Comparison between the Positions of the Giza Pyramids and Those of Orion Belt Stars}

A very famous archaeological site where the astronomical knowledge of the Egyptians, joint to their religious credence, could have played a relevant role in the monument building is the pyramid complex located in the Giza plain. As a significant example of this important interconnection, Spence (2000) and Belmonte (2001) have proposed that the stars of the constellations of Ursa Major and Ursa Minor were used to align to North the pyramids of this site, as well as of other ones. But the three main pyramids of Giza are also the subject of a suggestive and very controversial theory proposed by Bauval \& Gilbert (1994), the so-called "Orion Correlation Theory" (OCT). According to these authors, a perfect coincidence would exist between the mutual positions of the three stars of the Orion Belt and those of the main Giza pyramids, so that the latter would represent the monumental reproduction on the ground of that important asterism (see Bauval, 2006).

In a preliminary work (Orofino, 2014) the whole question was reanalyzed, subjecting the OCT to some quantitative astronomical and astrophysical verifications, in order to assess the compatibility of this theory with the results of both naked-eye astrometry and photometry. In the present paper, we summarize the previous results, subjecting the OCT to statistical verifications, in order to assess the probability that the correlation between stars and pyramids, both in terms of relative position and luminosity, can be merely casual.

To compare the positions of the Giza pyramids with those of the Orion Belt stars, we obtained, by means of the astronomical software Cartes du Ciel (downloadable at http://www.ap-i.net/skychart/it/start), a stellar map of that region of the sky relative to $2550 \mathrm{BC}$, the date around which one can assume the main pyramids of Giza were built (see i.e. Belmonte, 2001). The map has been conveniently rescaled (scale factor of $0.003^{\circ} / \mathrm{m}$ ) and rotated (anti-clockwise rotation of $195.3^{\circ}$ ) and has been overlapped to the topographic map of the Giza necropolis in order to minimize the star-pyramid distances, as shown in Figure 1. This figure shows that a certain discrepancy exists between the actual position of the vertex of each pyramid and the position expected on the basis of the stellar correlation; such a difference is more pronounced in the case of the couple Khufu-Alnitak where it is equal to $3.1 \%$ of the angular distance between Alnitak and Alnilam; since the angular separation of the two stars is $1.356^{\circ}$, this corresponds to about $2.5^{\prime}$.

This value is less than the resolution power of the human eye (defined as the minimum angular distance between two point sources necessary to see them as distinct objects); the latter in general falls between 5' and 10' 




Figure 1. Comparison between the positions of the Orion Belt stars (red dots) and those of the vertices of the corresponding pyramids. The star map (scale in red) has been overlapped to the topographic map of the Giza necropolis (scale in black with the related coordinate grid in grayafter Wakefield Sault, 2008). The crosses represent the error bars on the stellar positions and indicate the minimum uncertainty, equal to \pm 3 ', due to the resolution power of the human eye under optimal observation conditions (see text).

(Silvestro, 1989), according to the characteristics of the observed sources, and, in the most favorable cases, can be as low as 3' (Herrmann, 1975; Gribbin \& Gribbin, 1996). Any single measurement of stellar position (astrometry), performed with unaided eye, can never have an uncertainty less than this resolution power, and in general the former is much greater than the latter. For this reason it is very reasonable that a star map drawn with naked eye, such that the Egyptians would have used to reproduce on the ground the asterism of the Belt, could have an uncertainty on the stellar positions of the order of this physiological limit of the eye. Therefore the errors made by the pyramid builders in the positioning of these monuments, supposing they really wanted to represent the asterism of the Orion Belt, fall within the uncertainty range of the stellar positions known at that time. In other words, according to Bauval \& Gilbert (1994), the positions of the main pyramids of the Giza plain correspond to those of the Orion Belt stars within the error margin of the astrometric measurements of that epoch. This conclusion is possible without invoking the error of the Egyptian engineers to build the pyramids where the latter were planned. Taking into account also this error the star-pyramid compatibility is even stronger.

It is interesting to recall that, to overlap the Orion Belt stars on the Giza pyramids, it is first of all necessary to rotate of $180^{\circ}$ the celestial map, in the sense that the northernmost star (Mintaka) corresponds to the southernmost pyramid (Menkhaure). This apparent reversal of the North-South axis, however, does not constitute a problem at all, if , as suggested by Bauval (2006), the ancient Egyptians drew their geographic maps with South "at the top", so that Mintaka, the highest star of the Belt when the asterism culminates (reaches its highest point in the sky), was associated to the Menkhaure pyramid, the topmost one in the hypothetical topographic maps of 
that time, while Alnitak, the lowest star of the Belt at its culmination, corresponded to the Khufu pyramid, the lowest one in the maps. Obviously this choice is opposite to that adopted by the cartographers of the XVII century who decided to put North on the top of their maps, a convention that we too continue to make today. In any case there is no objective reason to put necessarily North at the top of the geographic charts. All the orientations are possible; it is only a matter of conventions. For example in the Medieval map known as the Hereford Mappa Mundi (dating to XIII century) East is at the top. According to Bauval (2006), for ancient Egyptians it was more logical to put South, and not North, on the top of their maps. South was "up" since the Nile River flows down from South and since the Sun culminates exactly in the South at midday. Actually, the Egyptians called (and we still call) the southern part of their country as "Upper Egypt" and the northern one as "Lower Egypt".

As reported above, an additional anti-clockwise rotation of $15.3^{\circ}$ is required to overlap the Orion Belt stars on the corresponding pyramids. Actually, such a rotation is necessary since the "axis of the Pyramids" (i.e. the straight line that best fits the positions of the centers, or vertexes, of the three Giza pyramids) is tilted of about $38^{\circ}$ with respect to the North-South direction, while the "axis of the Orion Belt" (the straight line that best fits the positions of the stars of the asterism) is tilted of about $53^{\circ}$ with respect to the celestial North-South direction. Therefore it is necessary a $15.3^{\circ}$ anticlockwise rotation of the star map in order to superimpose the two axes within the errors.

The different inclinations of the axes of the pyramids and of the Orion Belt with respect to the corresponding North-South direction (terrestrial, in the first case, celestial in the second one) can be simply due to religious motivations. Actually the south-eastern corners of the three Giza pyramids are aligned in good approximation towards the great solar temple of Heliopolis (Lehner, 1985a, b; Goedicke, 2001; Magli, 2009a, b). More precisely the straight line connecting the south-eastern corners of the two extreme pyramids (those of Menkaure and Khufu) passes about $12 \mathrm{~m}$ away from the corresponding corner of the central (Khafre) pyramid; however, since this discrepancy is less than $2 \%$ of the distance $(637 \mathrm{~m})$ between the corners of the extreme pyramids, it is reasonable to conclude that the disposition of the main pyramids on the Giza plain was likely dictated by religious considerations. In any case, it is worthwhile to note that obviously the Giza diagonal does not give constraints to the relative distance between pyramids. In other words this alignment towards Heliopolis does not necessarily imply that the pyramid had to be placed on the ground as we see them today: there are endless possibilities to position three pyramids in a way that respects the alignment of their south-eastern corners along a stray line. For this reason the question of the orientation of the pyramid axis is unessential for our goals (and in fact the test performed in the present work is insensitive to this parameter).

\section{Comparison between the Dimensions of the Giza Pyramids and the Magnitudes of the Stars of the Orion Belt}

In order to weigh up in detail the OCT, it is necessary to verify if the dimension of the pyramids is correlated with the brightness of the Orion Belt stars. The observed brightness of an object is measured by the so-called visual magnitude, defined by the Pogson formula:

$$
m=a \log F+m_{0}
$$

where $F$ is the flux (energy per unit time and area) of the visible radiation received by the observer, $a$ is constant equal to -2.5 , while $m_{0}$ is another constant conventionally chosen by assigning to a reference-star a $a$ priori fixed magnitude (the original choice was to use as a reference the Polar Star, assigning to it a visual magnitude $m=2$ ). By fixing in this way the two constants $a$ and $m_{0}$, Pogson made the modern star magnitude scale consistent with the ancient photometric classification of the stars performed in the II Century BC by Hipparchus. For the same historical reason, magnitude decreases with increasing flux (i.e. the brightest objects have the lowest magnitudes).

The visual magnitude of the Orion Belt stars is reported in Table 1. Since both Mintaka and Alnilam are variable stars, for them we used the average magnitudes reported, along with the magnitude of Alnitak (which is constant in time), in the recent Catalogo de Magnitudes Aparentes (Catalogue of Apparent Magnitudes-Otero, 2015). This catalogue, which will be completed shortly, provides for the first time combined information about the variability and the moltiplicity of all the stars with a visual magnitude less than 5 . Note that the magnitude of the three stars of the Belt are in good agreement with those reported in the work by Hardie, Heiser, \& Tolbert (1964) which is the most recent peer-reviewed paper on the subject. 
Table 1. Original values of the side of base $(l)$, the height $(h)$ and the volume $(V)$ of the three Giza pyramids, compared with the visual magnitude $(m)$ of the three stars of the Orion Belt (Otero, 2015). It is also reported the apparent height ( $h$ ') of each pyramid, that is the height of the vertex with respect to the base level of Khufu pyramid (see text).

\begin{tabular}{|c|c|c|c|c|c|c|}
\hline Pyramid & $l(\mathrm{~m})$ & $h(\mathrm{~m})$ & $h^{\prime}(\mathrm{m})$ & $V\left(\mathrm{~m}^{3}\right)$ & Star & $m$ \\
\hline Menkaure (Mykerinos) & 104 & 66 & 76 & $2.38 \times 10^{5}$ & Mintaka ( $\delta$ Orionis) & 2.23 \\
\hline Khafre (Chephren) & 215 & 144 & 154 & $2.22 \times 10^{6}$ & Alnilam ( $\varepsilon$ Orionis) & 1.69 \\
\hline Khufu (Cheops) & 230 & 147 & 147 & $2.59 \times 10^{6}$ & Alnitak ( $\zeta$ Orionis) & 1.76 \\
\hline
\end{tabular}

NOTE: The dimensions of the pyramids of Menkaure, Khafre and Khufu have been taken respectively from the sites: https://en.wikipedia.org/wiki/Pyramid_of_Menkaure, https://en.wikipedia.org/wiki/Pyramid_of_Khafre, https://en.wikipedia.org/wiki/Great_Pyramid_of_Giza

We have searched for a correlation between the visual magnitudes of the Orion Belt stars and the most obvious dimensions (that is the volume $V$ or the height $h$ ) of the corresponding pyramids. However no correlation has been found in these cases. The situation considerably changed when we considered, instead of the intrinsic height of each pyramid, the one that can be called "observed" height, $h$ '; the latter is the height of the pyramid vertex evaluated with respect to a common reference level, the same for the three pyramids, such as the lowest among the base levels of the three pyramids (see Table 1). This quantity (which, unlike the volume, is directly evaluable by only one measurement, also simply by eye) is exactly the one we take into consideration, for example, when we compare the heights of the mountains. Actually, the height that matters for an observer who looks at the pyramids from a distance is the observed height, that does not coincide with the intrinsic one, since the pyramids of Menkaure and Khafre are built on a plain at about $70 \mathrm{~m}$ on sea level, while the base of Khufu pyramid is located about $10 \mathrm{~m}$ lower down (Lehner, 1985a).

A plot of the visual magnitude $m$ of the Orion Belt stars versus the observed height $h$ ' of the corresponding pyramids with respect to the base level of the Khufu pyramid shows a significant anticorrelation between the two quantities (see Figure 2). Such an anticorrelation means that equal increases in $h$ ' correspond to equal decreases in $m$ and therefore to equal increments in the apparent brightness of the stars. The ancient Egyptian well knew this kind of geometric-mathematical relationship that they used many times when, for example, they planned and carried out architectural structures with constant slope (where equal horizontal displacements correspond to equal vertical displacements), such as the same pyramids and the shafts and the corridors inside them.

This result removes one of the most serious objections to the OCT, which is the claimed lack of correlation between the size of Giza pyramids and the brightness of the Orion Belt stars, while it is in agreement with the qualitative conclusions of Bauval \& Gilbert (1994).

\section{Statistical Verification of the OCT}

One of the most obvious questions that one can ask is whether the above discussed correlations between stars and pyramids, both in terms of relative position and luminosity/height, can be merely due to the case. To address this point the probabilities of random compatibility have been estimated, as it follows, by means of Monte Carlo simulations.

\subsection{Star Positions}

It is possible to characterize univocally the position of the three stars by means of two well measured parameters. The first one is the ratio $(\rho)$ between the Alnitak-Alnilam angular distance and the Alnilam-Mintaka angular distance: $\rho=1.0219$. The choice of the ratio is intuitive, because we must compare astronomical angular distances with terrestrial distances and the ratio is independent on measurement units. The second parameter is the angle $(\alpha)$ between the Alnitak-Alnilam axis and the Alnilam-Mintaka axis: $\alpha=172.483^{\circ}$. These two quantities are calculated starting from the star positions while the associated errors are evaluated assuming the uncertainties of the Egyptian measurements. Assuming a maximum error of $3^{\prime}=0.05^{\circ}$ in the star positions (see discussion in Sect. 2), we estimate $\rho=1.022 \pm 0.037, \alpha=172.5^{\circ} \pm 2.1^{\circ}$, where the errors are standard deviations. The errors on the star position have been propagated to $\rho$ and $\alpha$ by means of a standard computational method. 


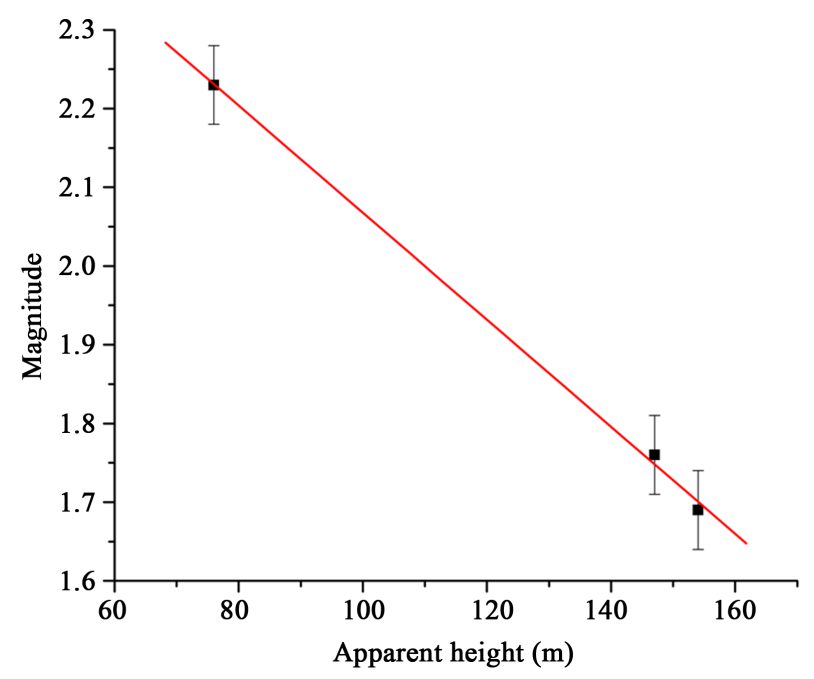

Figure 2. Straight line of linear regression between the apparent height ( $h$ ') of the pyramids and the visual magnitude $(\mathrm{m})$ of the corresponding stars of the Orion Belt (the error bar is due to the typical uncertainty of the evaluation by naked eye, equal to \pm 0.05 - Otero \& Moon, 2006). The linear anticorrelation between $h$ ' and $m$ implies a linear correlation between $h$ ' and the apparent brightness of the Orion Belt stars, that is a linear grow of the latter with the apparent height of the corresponding pyramids.

\subsection{Star Magnitudes}

As reported in Table 1, the magnitudes of the stars are $m_{1}=1.69$ (Alnilam), $m_{2}=2.23$ (Mintaka) and $m_{3}=1.76$ (Alnitak) with the same error (standard deviation $=0.05$, the typical uncertainty of the evaluation by naked eye-Otero \& Moon, 2006). Also in this case we use ratios in order to compare different quantities (star magnitude and pyramid observed height)

$$
\rho_{21}=m_{2} / m_{1}=1.320 \pm 0.069, \rho_{31}=m_{3} / m_{1}=1.041 \pm 0.060
$$

To complete the Orion Belt features the lowest magnitude/highest observed height must be associated with the star/pyramid in the vertex of angle $\alpha$. We neglect the association of different magnitudes with different angular distances because the $\rho$ ratio is compatible with 1, or rather may be that the ancient Egyptians consider the Alnilam-Mintaka angular distance equal to the Alnitak-Alnilam one. In other words the configuration Alnitak-Alnilam-Mintaka was not distinguishable from the configuration Mintaka-Alnilam-Alnitak.

\subsection{Simulation and Analysis}

For each pyramid three quantities have been randomly extracted from uniform distributions: distance $r$ and observed height $h^{\prime}$, both in the range $0-1$ (arbitrary units), and angle $\varphi$ in the range $0^{\circ}-360^{\circ}$ (see Figure 3 ). Therefore the positions are univocally determined in a circumference of unitary radius. We tested also the random position extraction on different surfaces (rectangles) and we got lower probabilities that the pyramid position is just a case. Only very asymmetric rectangles (at least with a side 4 times longer than the other one) allowed slightly higher probabilities but this particular geometry is not justified. In order to be conservative we chose the circular surface and generated 10 million of 3-pyramids configurations.

Four selection cuts have been applied to the 3-pyramids configurations (Figure 4) to estimate the percentage of those compatible with the Orion Belt. The cuts are reported in the Table 2 with two possible results (compatibility in 1 or 3 standard deviations). Each probability is the result of the associated cut and the previous ones, the probability errors are negligible because the simulated sample is very large. In both cases the final probability is very low. The more conservative probability (compatibility in 3 standard deviations) is only $0.018 \%$.

We recall that our approach implicitly assumes the possibility of a random distribution of pyramids on the terrain, regardless of topographic constraints, and this is clearly an oversimplification of the problem. However, as we reported above, our simulations have been performed with various shapes of the surface (circles, squares, 


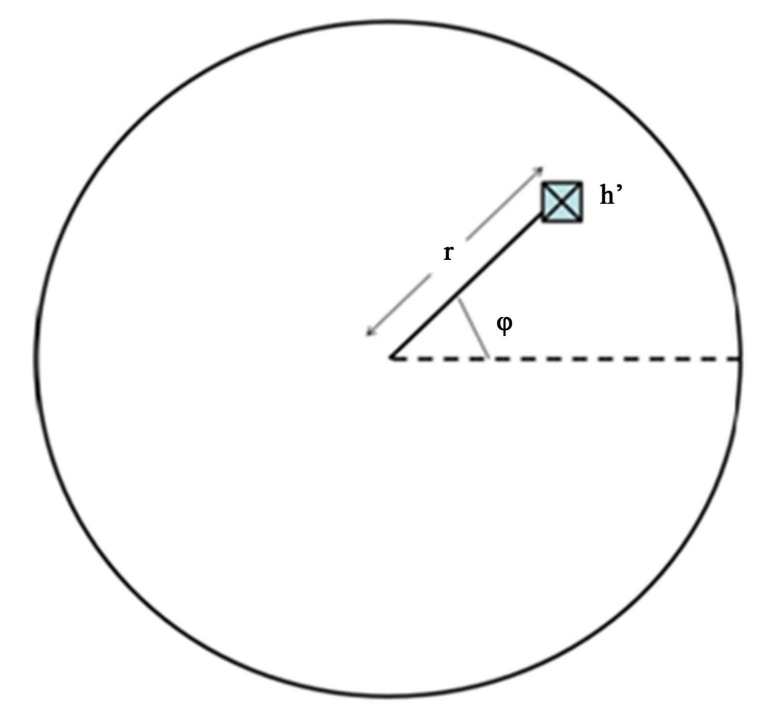

Figure 3. For each pyramid three quantities ( $r, \varphi$ and $h^{\prime}$ ) are randomly extracted from uniform distributions. Distance $r$ and angle $\varphi$ determine univocally the position in a circumference. The quantity $h$ ' represents the apparent height.

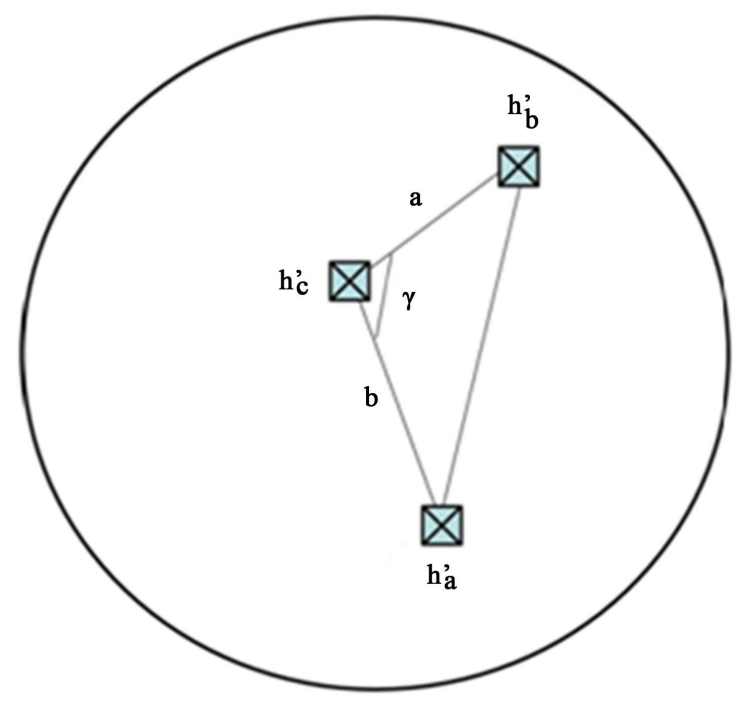

Figure 4. The simulated positions of the pyramids are studied looking at the value of the angle $\gamma$ and at the ratio between the distances ( $\mathrm{a} / \mathrm{b}$ or $\mathrm{b} / \mathrm{a})$. Also requirements about the height $\left(h_{\mathrm{a}}{ }^{\prime}, h_{\mathrm{b}}{ }^{\prime}\right.$ and $\left.h_{\mathrm{c}}{ }^{\prime}\right)$ are used (see text).

Table 2. Selection cuts applied to the randomly generated 3-pyramids configurations and their results (see Figure 4 for the meaning of the symbols). The second column reports the probability associated to a compatibility in 1 standard deviation, while the third column concerns the probability associated to a compatibility in 3 standard deviations.

\begin{tabular}{ccc}
\hline Selection cuts & $\mathrm{n}=1$ & $\mathrm{n}=3$ \\
\hline Angle: $|\gamma-\alpha|<2.1^{\circ} \mathrm{n}$ & $2.883 \%$ & $8.693 \%$ \\
\hline Distance ratio: $|\mathrm{a} / \mathrm{b}-\rho|<0.037 \mathrm{n}$ OR $|\mathrm{b} / \mathrm{a}-\rho|<0.037 \mathrm{n}$ & $0.143 \%$ & $0.935 \%$ \\
Highest pyramid in the $\gamma$ vertex: $h_{c}{ }^{\prime}>h_{a}{ }^{\prime}$ AND $h_{c}{ }^{\prime}>h_{b}{ }^{\prime}$ & $0.049 \%$ & $0.316 \%$ \\
Height ratio: $\left(\left|h_{c}{ }^{\prime} / h_{b}{ }^{\prime}-\rho_{21}\right|<0.069 \mathrm{n}\right.$ AND $\left.\left|h_{c}{ }^{\prime} / h_{a}{ }^{\prime}-\rho_{31}\right|<0.060 \mathrm{n}\right)$ & & $0.018 \%$ \\
OR & & $0.001 \%$ \\
\hline
\end{tabular}


rectangles) over which the pyramids have been distributed, finding results that are nearly independent on the shape. Furthermore, Egyptians were not strongly limited by the topographic constraints, since in building the pyramids they filled and leveled large parts of the Giza plain (Lehner, 1985a). In other words it seems that the exact location of the pyramids on the plain should not be dictated by topographic constraints, and for this reason our simulation approach of a random distribution of pyramids on the terrain represents a viable tool of analysis.

\section{Comparison between the Positions of the Giza Pyramids and Those of the Cygnus Stars}

Since, as discussed in the previous sections, our tests were not able to falsify the OCT, we checked the falsifying capability of our procedure by applying it to another very popular and controversial hypothesis, concerning the link between Giza pyramids and the stars: the so-called "Cygnus-Giza Correlation” (Collins, 2006). According to this theory, a perfect coincidence would exist between the mutual positions of the main Giza pyramids and those of the three stars ( $\varepsilon$ Cygni or Gienah, $\gamma$ Cygni or Sadr and $\delta$ Cygni) of the "wings" of the Swan, that is the stars which constitute the short arm of the asterism of the Northern Cross in the Cygnus constellation.

Following the same procedure reported in Section 2, the map of the Cygnus constellation relative to the present epoch has been overlapped to the topographic map of the Giza necropolis obtaining the fit shown in Figure 5(a). First of all, we note that in a circle centered around the star ( $\gamma$ Cygni) at the center of the Northern Cross, with radius equal to the angular distance between $\gamma$ Cygni and $\delta$ Cygni, there are not only the three stars, putative celestial counterparts of the Giza pyramids, but also two other stars, with a visual magnitude comparable with that of the group, that have no counterparts in the Giza plain; one of these is Deneb ( $\alpha$ Cygni), which is even the brightest of the constellation and is not associated to any ancient monument of the plain. At first glance, comparing Figure 5(a) with Figure 1, the mutual positions of the three stars of the short arm of the Northern Cross seem to fit, better than the stars of the Orion Belt, those of the vertices of the Giza pyramids. And this apparently very good correspondence is probably the reason of the popularity of the Cygnus-Giza Correlation.

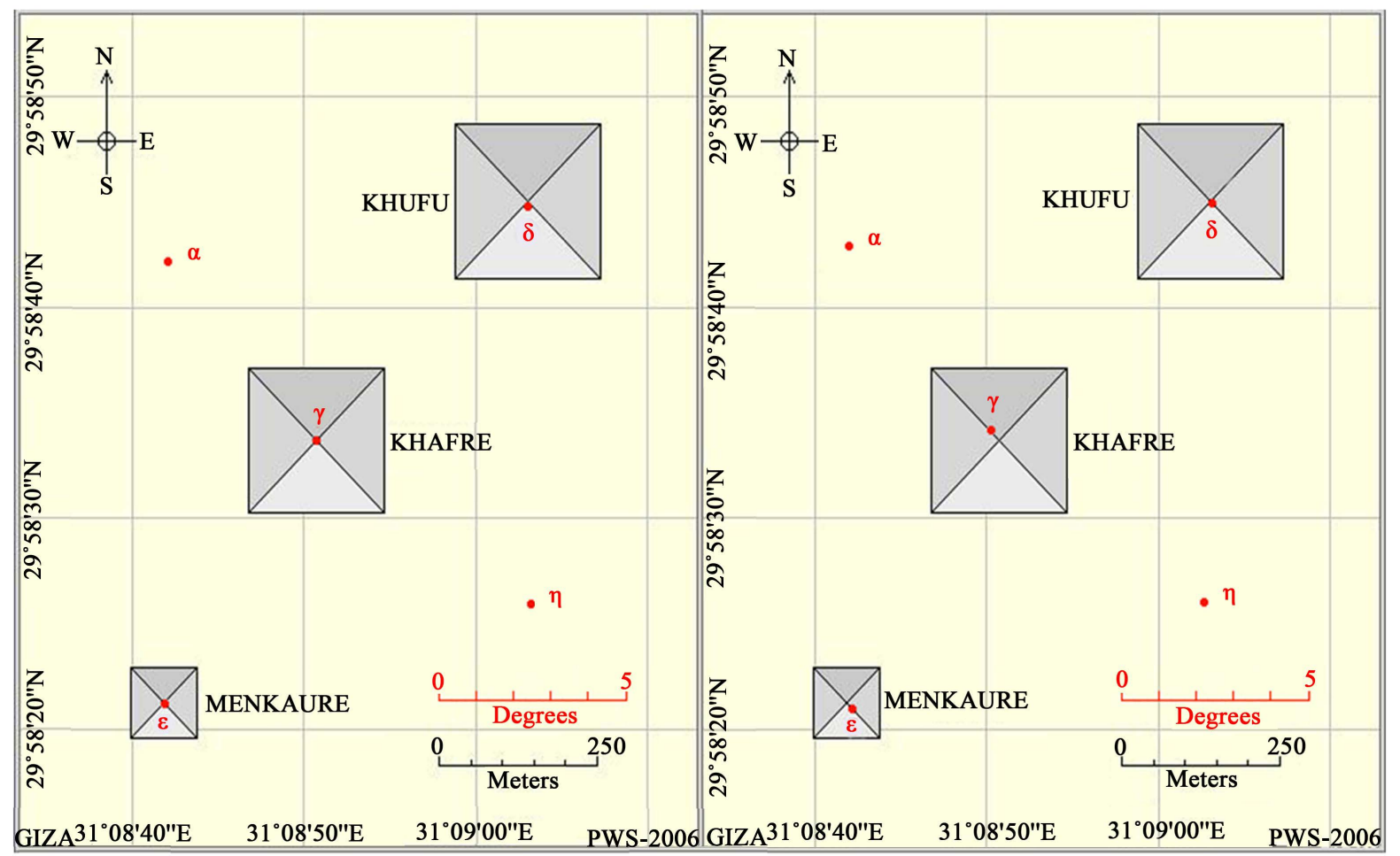

(a)

(b)

Figure 5. Same as in Figure 1 but in the case of the Cygnus stars for two epochs: (a) the present one; (b) the epoch of the presumed construction of the pyramids (2550 BC). Note that the errors on the star position are comparable with (but smaller than) the size of the dots representing the various stars. 
However, it is important to note that, due to the large angular dimension of the former asterism (about $16^{\circ}$ against less that $3^{\circ}$ for the Orion Belt), the uncertainty on the stellar positions $\left(\Delta=3^{\prime}=0.05^{\circ}\right.$-see Section 2) is comparable with the size of the dots which represent in Figure 5 each star of the Cygnus constellation. For this reason, if we consider the error on the star position, for these objects the fit is slightly worse than in the case of the Orion Belt. The fit worsens dramatically (see Figure 5(b)) when one considers the positions of the Cygnus stars around $2550 \mathrm{BC}$, that are sensibly different from the present ones, essentially due to the high proper motion of $\varepsilon$ Cygni. Actually, quantifying the quality of the fit by means of the $\chi^{2}$ parameter (Taylor, 1982):

$$
\chi^{2}=\sum_{i}\left(\frac{d_{i}}{\sigma}\right)^{2}
$$

(with $d_{i}$ discrepancies of the stellar positions from the vertices of the pyramids and $\sigma$ their standard deviation), and assuming $\sigma=\Delta$, the fit shown in Figure 5(b) is characterized by the value $\chi^{2}=50$, while for the fit shown in Figure 1 one has $\chi^{2}=2$. In particular, in order to obtain for the Cygnus-Giza case a $\chi^{2}$ value comparable to the one obtained for the Orion Belt-Giza case is necessary to assume an error $\Delta=15^{\prime}$, that is five times greater than that assumed for the latter case (see above). Therefore, the relative positions of $\varepsilon, \delta$ and $\gamma$ Cygni reproduce the mutual positions of the Giza pyramids much worse than the Orion Belt stars. Furthermore, it must be taken into account that, in the fit obtained with the Cygnus stars, the star of medium brightness ( $\varepsilon$ Cygni) is associated with the Menkaure pyramid which is the smallest one, irrespective of the considered dimension (volume, intrinsic height, observed height), while the dimmest star ( $\delta$ Cygni) is associated with the Khufu pyramid that, whatever the considered dimension, is the largest one. In other words there is no correlation between the magnitude of the Cygnus stars and none of the plausible dimensions (volume, intrinsic height, observed height) of the pyramids.

This lack of correspondence between Cygnus stars and Giza pyramids, both in relative position and in brightness/dimension, is in contrast with the Cygnus hypothesis and, for this reason, the statistical approach described in Section 4 for OCT is clearly not applicable to this case.

\section{Discussion and Conclusion}

The results found in the previous sections can be summarized as follows: a) the relative positions of the three Giza pyramids coincide, within the uncertainties of the naked-eye astrometric measurements, with the relative positions of the three stars of the Orion Belt; b) the visual magnitude of the stars of the Belt is presently correlated with the height of the corresponding pyramids evaluated with respect to a common reference level (e.g. the base level of the Khufu pyramid); c) using a simple Monte Carlo simulation, the probability that the correspondence between the Giza pyramids and the Orion Belt is just due to the case, has been estimated to be very low (less than $0.02 \%$ ).

Since the star evolution models suggest that the magnitudes of all the three objects of the Belt at the time of the pyramids were substantially equal to the present ones, the above found correlation was still valid at that epoch (Orofino, 2011).

In the light of the previous results, one can conclude that the OCT is compatible with what expected for the stars of the Orion Belt on the basis of naked-eye astrometry and photometry, as well as of the stellar evolution theory. Furthermore, it is unlikely that the above cited correlation can be fortuitous. Therefore, there are no astronomical/astrophysical and statistical arguments to reject the hypothesis that the main Giza pyramids will represent the monumental reproduction on the ground of the Orion Belt (Bauval \& Gilbert, 1994; Bauval, 2006).

After this failure of our approach in falsifying the OCT, we have subjected to the same astronomical tests also another group of stars, searching for their presumed correlations with the Giza pyramids; in particular we have considered the three stars of the short arm of the Northern Cross, in the Cygnus constellation. In this case, however, unlike what stated by the Cygnus-Giza Correlation (Collins, 2006), we have found no correspondence between the above mentioned stars and the main pyramids of Giza, both in relative position and, specially, in brightness/dimension and therefore this hypothesis is not supported by our tests.

\section{Acknowledgements}

The authors warmly thank Sebastian Otero and Chris Tedder for the very useful discussions on various subjects treated in the text that helped to improve the quality of the manuscript. Marcella D'Elia is also thanked for her technical assistance in preparing the printed version of the present paper. 


\section{References}

Bauval, R. G. (2006). The Egypt Code. London: Century Publishing.

Bauval, R. G., \& Gilbert, A. (1994). The Orion Mystery. Oxford: Heinemann.

Baux, N. (1994). Sirius étoile et jeune Horus. In C. Berger, G. Clerc, \& N. Grimal (Eds.), Hommages à Jean Leclant (pp. 61-72). Le Caire: Institut Français d'Archéologie Orientale, Biblioteque d'Etude 106/1.

Belmonte, J. A. (2001). On the Orientation of Old Kingdom Egyptian Pyramids. Journal for the History of Astronomy, 32, S1-S20. http://dx.doi.org/10.1177/002182860103202601

Belmonte, J. A., Shaltout, M., \& Fekri, M. (2008). On the Orientation of Ancient Egyptian Temples: (4) Epilogue in Serabit el Khadem and Overview. Journal for the History of Astronomy, 39, 181-211. http://dx.doi.org/10.1177/002182860803900203

Collins, A. (2006). The Cygnus Mystery. London: Watkins Publishing.

Goedicke, H. (2001). Abusir-Saqqara-Giza. In M. Barta, \& J. Krejci (Eds.), Abusir and Saqqara in the Year 2000 (pp. 397-412). Prague: AVCR.

Gribbin, J., \& Gribbin, M. (1996). Companion to the Cosmos. London: The Orion Publishing Group.

Hardie, R. H., Heiser, A. M., \& Tolbert, C. R. (1964). A Study of the B Stars in the Orion Belt Region. Astrophysical Journal, 140, 1472-1481. http://dx.doi.org/10.1086/148052

Herrmann, J. (1975). Atlante di Astronomia. Milano: Mondadori.

Legon, J. A. R. (1995). The Orion Correlation and Air-Shaft Theories. Discussions in Egyptology, 33, 45-56.

Lehner, M. (1985a). The Development of the Giza Necropolis: The Khufu Project. Mitteilungen des Deutschen Archaologischen Instituts Abteilung Kairo, 41, 109-143.

Lehner, M. (1985b). A Contextual Approach to the Giza Pyramids. Archiv für Orientforschung, 31, 136-158.

Magli, G. (2006). On the Possible Discovery of Precessional Effects in Ancient Astronomy. In R. Bauval (Ed.), The Egypt Code (Appendix 2). London: Century Publishing. http://arxiv.org/pdf/physics/0407108

Magli, G. (2009a). Akhet Khufu: Archaeo-Astronomical Hints at a Common Project of the Two Main Pyramids of Giza, Egypt. Nexus Network Journal, 11, 35-50. http://dx.doi.org/10.1007/s00004-008-0080-3

Magli, G. (2009b) Astronomy, Topography and Dynastic History in the Age of the Pyramid Fields of the Old Kingdom. http://arxiv.org/abs/0903.1416

Orofino, V. (2011). Stato evolutivo delle stelle della Cintura di Orione ed implicazioni archeoastronomiche. English Version in Preparation. http://arxiv.org/abs/1109.3284

Orofino, V. (2014). A Quantitative Astronomical Analysis of the Orion Correlation Theory. http://arxiv.org/abs/1109.6266

Otero, S. A. (2015). Catalogo de Magnitudes Aparentes, in Preparation. (Requests Can Be Addressed to varsao@hotmail.com)

Otero, S. A., \& Moon, T. (2006). The Characteristic Period of Pulsation of $\beta$ Gruis. The Journal of the American Association of Variable Star Observers, 34, 156-164.

Silvestro, G. (1989). Astronomia. Torino: La Scientifica Editrice.

Spence, K. (2000). Ancient Egyptian Chronology and the Astronomical Orientation of Pyramids. Nature, 408, 320-324. http://dx.doi.org/10.1038/35042510

Taylor, J. R. (1982). An Introduction to Error Analysis. Mill Valley, CA: University Science Books.

Wakefield Sault, P. (2008). The Keys of Atlantis. A Study of Ancient Unified Numerical and Metrological Systems. Appendix C. http://www.odeion.org/atlantis/appendix-c.html 